\title{
Plasma Cell-free DNA 5-Hydroxymethylcytosine and Whole-Genome Sequencing Signatures for Early Detection of Esophageal Cancer
}

Di Lu

Nanfang Hospital, Southern Medical University

Xuanzhen Wu

Nanfang Hospital, Southern Medical University

Shuangxiu Wu

Berry Oncology Corporation

Hui Li

Berry Oncology Corporation

Xuebin Yan

Nanfang Hospital, Southern Medical University

Jianxue Zhai

Nanfang Hospital, Southern Medical University

Xiaoying Dong

Nanfang Hospital, Southern Medical University

Siyang Feng

Nanfang Hospital, Southern Medical University

Fuming Sun

Berry Oncology Corporation

Shaobo Wang

Berry Oncology Corporation

Xueying Zhang

Berry Oncology Corporation

Kaican Cai ( $\square$ doc_cai@163.com )

Nanfang Hospital, Southern Medical University

\section{Research Article}

Keywords: esophageal cancer, early diagnosis, 5-hydroxymethylcytosine, low-passwhole-genome sequencing, cell-free DNA

Posted Date: March 2nd, 2022 
DOI: https://doi.org/10.21203/rs.3.rs-1375061/v1

License: (c) (1) This work is licensed under a Creative Commons Attribution 4.0 International License. Read Full License 


\section{Abstract \\ Background}

Esophageal cancer is one of globally high incidence and mortality disease. Its early stage has rarely obvious symptoms. Compared to conventional endoscopy diagnosis, liquid biopsy is an emerging noninvasive method for cancer early detection.

\section{Methods}

We enrolled 100 esophageal squamous cell carcinoma (ESCC) patients and 71 healthy individuals as a Southern China cohort and performed 5-hydroxymethylcytosine $(5 \mathrm{hmC})$ sequencing on their plasma cellfree DNA (cfDNA). A Northern cohort of cfDNA 5hmC dataset with 150 ESCC patients and 183 health individuals were downloaded for validation. A diagnostic model was firstly developed based on cfDNA $5 \mathrm{hmC}$ signatures and then improved by low-pass whole genome sequencing (WGS) features of cfDNA.

\section{Results}

Conserved cfDNA $5 \mathrm{hmC}$ modification motifs were observed in the two independent ESCC cohorts. A diagnostic model with $2735 \mathrm{hmC}$ features based on randomly-selected two-thirds samples of the Southern China cohort was validated independently in the left one-thirds samples and the whole Northern China cohort, achieved an AUC of 0.810 and 0.862 with sensitivities of $69.3-74.3 \%$ and specificities of 82.4-90.7\%, respectively. The performance was well maintained in Stage I to Stage IV, with accuracy of $70 \%-100 \%$, but low in Stage 0, with accuracy of 33.3\%. Low-pass WGS of cfDNA improved the AUC to 0.934 with a sensitivity of $82.4 \%$, a specificity of $88.2 \%$ and an accuracy of $84.3 \%$, particularly significantly in Stage 0 with the accuracy up to $80 \%$.

\section{Conclusions}

This study suggests that the blood-based 5hmC integrated with low-pass WGS model could improve the accurate diagnosis of early stage ESCC, particularly for very early stage ESCC.

\section{Introduction}

Esophageal cancer is a global problem threatening people's health and declining people's life expectancy which ranks the seventh in terms of incidence and the sixth in mortality worldwide in 2020 with two main subtypes: esophageal squamous cell carcinoma (ESCC) and esophageal adenocarcinoma (EAC)[1].There are distinct geographic variations between the two main subgroups of esophageal cancer. The incidence of ESCC is apparently higher in China, Central Asia, East and South of Africa while most of the EAC cases occur in patients living in high income countries like North America and Europe[2]. Most of the patients 
were already suffered from ESCC with an advanced stage when they sought medical advices as early esophageal cancer was rarely accompanied by distinct symptoms, thus, neither the surgical procedures nor adjuvant therapies could lead to satisfied outcomes[3]. So, an urgent demand for a new method to help making early diagnosis of esophageal carcinoma is desired.

Generally, tissue biopsy like endoscopy is the most widespread procedure used to make detection of esophageal cancer and help formulating stages for further therapeutic schedule. However, there remains obvious shortcomings and inadequacies like inferior diagnostic efficiency, uncomfortable checking process and expensive spending which may lead to poor adherence to subsequent treatments in return[4, 5]. Compared to conventional tissue biopsy, liquid biopsy is an emerging non-invasive method to make early detection which can also make further molecular and genic analysis using these liquid specimens[6]. Thereinto, cell-free DNA (cfDNA) is deliberated from the cell existing in blood, lymph, bile, milk, urine, saliva, mucous suspension, spinal fluid and amniotic fluid originating from apoptosis, necrosis, phagocytosis, nocosis and active secretion[7], and was proved to be a approving biomarker to make screening, detection and monitoring of cancers[8]. However, there remains some challenges in the application of cfDNA for early detection of cancers like hyposecretion, variability and polyphyly[9]. The efficiency of DNA methylation as an epigenetic mark has been previously validated[10], furthermore, the association between DNA methylation and tumorigenesis has been verified too[11, 12]. As the conversion of cytosine, 5-hydroxymethylcytosine $(5 \mathrm{hmC})$ is oxidized from 5-methylcytosine $(5 \mathrm{mC})$ by the enzyme teneleven translocation 1(TET1) which is one of the three enzymes of TET(ten-eleven translocation) family and is recognized as a better marker to detect gene expression[13]. Although there are fewer $5 \mathrm{hmC}$ existing in tissues and more challenges to accomplish precise detection, it has been proved that $5 \mathrm{hmC}$ has a superior permissive effect on gene expression and exhibits more tissue specificity. Besides, plenty of studies have reported that $5 \mathrm{hmC}$ in cfDNA was used as a promising marker to detect cancers like earlystage pancreatic cancer, non-small-cell lung cancer (NSCLC), hepatocellular carcinoma (HCC), blood and colon cancer[14-19]. In addition, a recent study showed ctDNA in plasma had distinct features, such as shorter fragment size, special motif and nucleosome footprint (NF) in comparison with cfDNA of healthy people[20], which could be applied to an advanced technology (low-pass whole-genome sequencing (WGS)) to get more accurate outcomes in identifying ctDNA from plasma. WGS of plasma cfDNA was a new empowering technology used for non-invasion low-disease-burden diagnosis. This technology provided ultra-sensitive detection of ctDNA from cfDNA of healthy individuals via genome-wide mutational integration[20]. Recent studies proved its good performance on early diagnosis of lung cancer[21, 22], hepatocellular carcinoma (HCC)[23] and malignant peripheral nerve sheath tumor (MPNST)[24].

Therefore, employing $5 \mathrm{hmC}$ in plasma cfDNA as a biomarker to distinguish the differences between ESCC patients and healthy control people $(\mathrm{HC})$ is worth trying, and we aimed to determine $5 \mathrm{hmC}$ signatures in cfDNA using machine learning methods as early diagnostic biomarkers for ECSS in a prospective cohort study. The low-pass WGS technology was also attempted as a complementary technique for identification of early ESCC from healthy people. 


\section{Results}

\section{Samples composition and study design}

A total of 150 adult subjects were prospectively enrolled in this study from March 2019 to December 2020, including patients with Early ESCC (stages $0, I A$ and IB, $n=50$ ), and middle and advanced (Mid-Ad) ESCC (stages II, III and IVA, $n=50)$ and healthy control individuals $(H C, n=50)$ from Nanfang Hospital of Southern Medical University, as a Southern ESCC cohort since the patients were mainly from the South of China. Detailed clinical information regarding gender, age, BMI, living habits, differentiation of cancer, TNM stages and surgery selections were illustrated in Table 1. And after conducting a brief statistical analysis using the data of the 150 patients collected from our hospital, we observed that there were no apparent differences among most of the variates like gender, age, BMI, habits and the incidences of hypertension and diabetes $(P>0.05)$, except for family history $(P<0.05)$, which suggested that family history might play an important role in tumorigenesis. 
Table 1

Summary of demographic and clinicopathological characteristics of all the participants in this study

Characteristics

$\mathrm{HC}$

$(n=50)$

Early ESCC (n Mid-Ad ESCC

$P$

$(n=50)$

value

\section{Demographic}

\begin{tabular}{|c|c|c|c|c|c|}
\hline \multirow[t]{2}{*}{ Gender } & Male & 37 & 37 & \multicolumn{2}{|l|}{37} \\
\hline & Female & 13 & 13 & 13 & \\
\hline \multicolumn{2}{|l|}{ Age (years) (mean, range) } & $\begin{array}{l}58(50- \\
70)\end{array}$ & $60(50-70)$ & $59(50-70)$ & 0.15 \\
\hline \multirow{2}{*}{\multicolumn{2}{|c|}{$\begin{array}{l}\text { BMI } \\
\text { (mean, range) }\end{array}$}} & 22.9 & 22.2 & 22.0 & 0.28 \\
\hline & & $\begin{array}{l}(15.2- \\
28.7)\end{array}$ & $(17.6-29.8)$ & $(16.4-27.3)$ & \\
\hline \multicolumn{2}{|l|}{ Salted foods (like/dislike) } & $13(29)^{*}$ & $16(34)$ & $25(25)$ & 0.106 \\
\hline \multicolumn{2}{|l|}{ Smoking (yes/no) } & $22(20)^{\star}$ & $30(20)$ & $31(19)$ & 0.667 \\
\hline \multicolumn{2}{|l|}{ Drinking (yes/no) } & $20(22) \star$ & $20(30)$ & $29(21)$ & 0.201 \\
\hline \multicolumn{2}{|l|}{$\begin{array}{l}\text { Fresh vegetables and fruits } \\
\text { (like/dislike) }\end{array}$} & $30(12)^{*}$ & $38(12)$ & $34(16)$ & 0.654 \\
\hline \multicolumn{2}{|l|}{ Family history (with/without) } & $0(42)^{*}$ & $10(40)$ & $6(44)$ & 0.005 \\
\hline \multicolumn{2}{|l|}{ Hypertension (with/without) } & $7(35)^{\star}$ & $13(37)$ & $7(43)$ & 0.282 \\
\hline \multicolumn{2}{|l|}{ Diabetes (with/without) } & $1(41)^{\star}$ & $6(44)$ & $5(45)$ & 0.212 \\
\hline \multicolumn{6}{|l|}{ Clinical } \\
\hline \multirow[t]{4}{*}{ Differentiation } & G0 & NA & 27 & NA & \\
\hline & G1 & NA & 16 & 20 & \\
\hline & $\mathrm{G} 2$ & NA & 6 & 21 & \\
\hline & G3 & NA & 1 & 9 & \\
\hline \multirow[t]{4}{*}{ TNM stages } & 0 & NA & 27 & NA & \\
\hline & IA & NA & 3 & NA & \\
\hline & IB & NA & 20 & NA & \\
\hline & $\| A$ & NA & NA & 19 & \\
\hline
\end{tabular}

Note: Patients were classified into 3 groups; HC, healthy controls; Mid-Ad, middle-advanced; ESCC, esophageal squamous cell carcinoma; MATHE: mediastinoscope-assisted transhiatal esophagectomy; ESD: endoscopic submucosal dissection; $\mathrm{P}$ value in chi-square test or $\mathrm{t}$ test. *There were 8 unknown findings. 


\begin{tabular}{|c|c|c|c|c|c|}
\hline \multicolumn{2}{|l|}{ Characteristics } & \multicolumn{2}{|l|}{$(n=50)$} & $\begin{array}{l}\text { Mid-Ad ESCC } \\
(n=50)\end{array}$ & $\begin{array}{l}P \\
\text { value }\end{array}$ \\
\hline & IIB & NA & NA & 7 & \\
\hline & IIIA & NA & NA & 1 & \\
\hline & IIIB & NA & NA & 19 & \\
\hline & IVA & NA & NA & 4 & \\
\hline \multirow[t]{5}{*}{ Surgery } & MATHE & NA & 12 & NA & \\
\hline & McKeown & NA & 9 & 24 & \\
\hline & Sweet & NA & 18 & 25 & \\
\hline & IvorLewis & NA & NA & 1 & \\
\hline & ESD & NA & 11 & NA & \\
\hline $\begin{array}{l}\text { Note: Patients w } \\
\text { esophageal squ } \\
\text { esophagectomy } \\
\text { were } 8 \text { unknown }\end{array}$ & $\begin{array}{l}3 \text { groups; } H \\
\text { oma; MATH } \\
\text { c submucos }\end{array}$ & $\begin{array}{l}\text { healthy } \\
\text { mediasti } \\
\text { dissectio }\end{array}$ & $\begin{array}{l}\text { ntrols; Mid-Ad, } \\
\text { scope-assisted } \\
\text { P value in chi-sc }\end{array}$ & $\begin{array}{l}\text { ddle-advanced; } \\
\text { anshiatal } \\
\text { lare test or t tes }\end{array}$ & $\begin{array}{l}\text { C, } \\
\text { here }\end{array}$ \\
\hline
\end{tabular}

Additional $21 \mathrm{HC}$ samples were acquired from a previously published study[25]. The HC cohort was identified as satisfying the study inclusion criteria, which were specifically negative for any form of cancer. Detailed pathological and clinical information was obtained from clinical records and illustrated in Table 1 and supplementary Table S1. The subject age and gender distributions among HC, Early ESCC and Mid-Ad ESCC groups were unbiased based on the Kruskal-Wallis $\mathrm{H}$-test ( $P$ value $=0.11$, supplementary Figure S1A) and Fisher test ( $P$ value $>0.5$, supplementary Figure S1B), respectively. Our primary aim was to develop a convenient and excellent diagnostic model using new biotechnology reflecting genome-wide signatures from plasma cfDNA to distinguish patients with Early ESCC from HC subjects. For biomarker screening and classifier model construction for early diagnosis of ESCC, the ESCC patients and $\mathrm{HC}$ individuals were randomly divided into two groups: about $2 / 3$ individuals as a training set and 1/3 individuals as an independent test set (also named as the internal test set) (Fig. 1 and supplementary Figure $\mathrm{S} 2$ ). We also downloaded $5 \mathrm{hmC}$-sequencing data from previously published studies of ESCC (as another independent external test set, also named as a Northern ESCC cohort since these ESCC patients were mainly from the North of China)[26].

\section{Conserved 5hmc Modification Changes In Escc, Potential Biomarkers For Diagnosis}

To explore the distribution patterns of hydroxymethylation in plasma cfDNAs across the genome, we first identified $5 \mathrm{hmC}$-enriched regions in each sample and subsequently determined as reliable peaks by high enrichment and significance as described in Methods. Comparison of global $5 \mathrm{hmC}$ modification levels 
between ESCC and $\mathrm{HC}$ groups revealed significant differences. The density of $5 \mathrm{hmC}$ peaks number were calculated and exhibited a broader distribution in ESCC group compared with the HC group that displayed a sharper and narrower curve (Fig. 2A). 5hmC peaks number in ESCC cfDNAs cohort was significantly more than that in $\mathrm{HC}$ cohort (Mann-Whitney U-test, $P$ value $=3.11 \times 10^{-5}$, supplementary Figure $\mathrm{S} 3$ ) and showed a gradually increasing trend from stage 0 to stage IV patients (Mann-Kendall Test, $P$ value $=1.65$ $\times 10^{-3}$, Fig. $2 \mathrm{~B}$ ), indicating that $5 \mathrm{hmC}$ modification changes might play an important role in promoting tumorigenesis and progression of esophagus cancer. In addition, increased $5 \mathrm{hmC}$ modification levels within promoter and genebody regions in ESCC group were observed by $5 \mathrm{hmC}$ metagene profile analysis (Fig. 2C), consistent with previous observation in ESCC[26]. To further compare the cfDNA 5hmC modification difference between the two groups, we determined differential $5 \mathrm{hmC}$ peaks with DESeq2 package ( $P$ value $<0.01)$ and identified $3985 \mathrm{hmC}$ up-regulation peaks and $2275 \mathrm{hmC}$ down-regulation peaks in ESCC groups by comparing with $\mathrm{HC}$ groups. $5 \mathrm{hmC}$ up-regulation peaks from ESCC patients were significant enriched in promoters $(28.14 \%)$ and 1 st intron regions $(15.58 \%)$ (i.e., mainly in the regulation regions of a gene) on the whole genome level, while more $5 \mathrm{hmC}$ down-regulation peaks were located in other introns (37.44\%) and distal intergenic regions (39.21\%) (Fig. 2D).

Next, $5 \mathrm{hmC}$ motif enrichment analysis was performed in up-regulation and down-regulation regions of $5 \mathrm{hmC}$ signals in ESCC samples to further understand the correlation of $5 \mathrm{hmC}$ changes with potential interactions of binding proteins. The $5 \mathrm{hmC}$ up-regulation peaks in ESCC was significantly enriched in ERG motif ( $P=1 \mathrm{e}-5,28.83 \%)$, followed by ETS1 ( $P=1 \mathrm{e}-4,20.25 \%)$ and ETV2 motif ( $P=1 \mathrm{e}-3,17.79 \%)$, all of which belong to the large family of ETS transcription factors and bind to the consensus DNA sequence 5 'AGGAA-3' (left in Fig. 2E), most of which are downstream nuclear targets of Ras-MAP kinase signaling, and associate with cell development, differentiation, proliferation, apoptosis and tissue remodeling. The deregulation of ETS genes results in the malignant transformation of cells and is often observed in various types of malignant tumors[27]. In contrast, the motif of GATA3 ( $P=1 \mathrm{e}-5,29.23 \%)$, GATA4 $(P=1 \mathrm{e}-$ $5,22.31 \%)$ and TRPS1 ( $P=1 \mathrm{e}-4,33.85 \%)$ were observed in 5hmC down-regulation peaks (right in Fig. 2E). GATA transcription factor mutations or dysregulated GATA expression were reported to associate with the wide range of diseases and pathologic phenotypes[28]. These are consistent with previously published study showing that ETS motif and GATA motif were identified in $5 \mathrm{hmC}$-gain and $5 \mathrm{hmC}$-loss regions for esophageal cancer, respectively[26]. On the other hand, we further compared the enriched $5 \mathrm{hmC}$ motifs among our Southern ESCC cohort, Northern ESCC cohort[26] and colorectal cancer cohort[25] and observed. For the top 10-significantly hit 5hmC motifs, about 30.0\%-50.0\% 5hmC motifs shared between the Southern and the Northern ESCC cohorts, obviously more than those shared with CRC patients (about 10.0\%-20.0\%) (Fig. 2F, supplementary Table S2). These results showed that the unique signature of plasma cfDNA $5 \mathrm{hmC}$ may represents a stable biomarker for discriminating ESCC and healthy individuals and could be potentially useful for early ESCC diagnosis.

\section{Screening, Validation And Performance Of Candidate 5hmc Biomarkers And Classifier}


Since the results of $5 \mathrm{hmC}$ up-regulation peaks were significantly enriched in promoter and $1 \mathrm{st}$ intron regions and $5 \mathrm{hmC}$ down-regulation peaks were significantly enriched in other introns and distal intergenic regions in the ESCC patients, which indicated that differential $5 \mathrm{hmC}$ signals in promoter and certain genebody regions might be utilized for identifying candidate $5 \mathrm{hmC}$ biomarkers to develop a convenient and stable diagnostic model. All 171 samples were randomly separated into two groups ( 54 HC, 34 Early ESCC and 31 Mid-Ad ESCC for training set, the remaining samples for internal test set) for development and evaluation of diagnostic model. The trained model was also validated on the independent previouslypublished Northern ESCC dataset (external test set) included 150 esophageal cancer and $183 \mathrm{HC}$ samples[26], which was profiled for $5 \mathrm{hmC}$ using a nano-hmC-Seal method similar to the one used in our study.

Firstly, 925 candidate $5 \mathrm{hmC}$ marker genes derived from promoter and genebody regions were selected by Wilcoxon rank-sum test $P$ values $<0.001$ in the training set. Using Recursive feature elimination - Cross Validation (RFECV) approach, we further identified a disease-specific panel of $2735 \mathrm{hmC}$ marker genes according to the contribution of each gene in the model (supplementary Table S3), and the distinct 5hmC landscapes in cfDNA showed apparent separation between ESCC and HC groups by unsupervised hierarchical clustering analysis (Fig. 3A). Similarly, the principal component analysis (PCA) also demonstrated distinct signatures that could discriminate the majority of the individuals of the two groups (Fig. 3B). To explore the biological significance of 273 marker genes with differential $5 \mathrm{hmC}$ signals, we performed gene ontology (GO) and Kyoto Encyclopedia of Genes and Genomes (KEGG) functional enrichment analysis and found that $273 \mathrm{5hmC}$ biomarkers were enriched in pathways associated with cancer and metastasis and mapped to tumor-related genes (Fig. 3C). For instance, Fig. 3D exhibited the IGV plot of the high-weight biomarker located at the FOXK1 gene, which plays an oncogenic role in the development of esophageal cancer[29]. The ESCC samples showed increased $5 \mathrm{hmC}$ modification level at FOXK1 gene when compared with the healthy controls. The classifier model based on 273 marker gene illustrated decent capacity for distinguishing ESCC patients from $\mathrm{HC}$ individuals in both internal test set Area under curve $(A U C)=0.810$ (95\% Cl: 0.693-0.927); sensitivity $=74.3 \%$; specificity $=82.4 \%$ ) and external test set (AUC $=0.862$ (95\% Cl: 0.822-0.902); sensitivity $=69.3 \%$; specificity $=90.7 \%$ ) (Fig. $3 \mathrm{E}$ and $3 \mathrm{~F})$. The prediction performance of classifier model in external test set was superior to that of internal test set, probably because of patients with 0 stage accounting for $27 \%(27 / 100)$ of our ESCC cohort who might be misclassified as $\mathrm{HC}$ individuals.

We next assess the prediction accuracy of $5 \mathrm{hmC}$ biomarker classifier for different clinical stages in our ESCC patient subgroups. Box plots exhibited that the probability of being predicted as cancer gradually increasing with the progression of cancer stage in the test set (Fig. 3G). The $5 \mathrm{hmC}$ score between Early ESCC (stage 0 and $\mathrm{I}$ ) and $\mathrm{HC}$ individuals demonstrated statistically significant disparity $(P$ value $=$ $4.35 \times 10^{-2}$, supplementary Figure $S 4$ ), which suggested the capacity of the $5 \mathrm{hmC}$ model to discriminate Early ESCC patients from $\mathrm{HC}$ individuals. Meanwhile, $5 \mathrm{hmC}$ score also accurately distinguished between stage I and $\mathrm{HC}$ samples $\left(P\right.$ value $\left.=4.54 \times 10^{-3}\right)$, stage 0 and stage I, II, III-IV $\left(P\right.$ values $=4.64 \times 10^{-2}$, $1.46 \times 10^{-2}$ and $9.85 \times 10^{-3}$, respectively, Fig. $3 \mathrm{G}$ ). However, in differentiating stage 0 from $\mathrm{HC}$ samples, 
$5 \mathrm{hmC}$ score was not the best diagnostic feature ( $P$ value $=0.40$, Fig. $3 \mathrm{G})$, and the diagnostic accuracy (33.3\%, supplementary Figure S5B) need to be further improved.

\section{Integrated model based on cfDNA signatures of low-pass WGS and $5 \mathrm{hmC}$ biomarkers improved diagnostic score for Early ESCC}

To explore the prediction potential of plasma cfDNA and search for more effective biomarkers, we employed low-pass whole-genome sequencing (WGS) to acquire genome-wide 5' end motif[30], nucleosome footprint (NF)[31] and fragmentation[8] profiles from $71 \mathrm{HC}$ and $93 \mathrm{ESCC}$ samples with enough plasma cfDNA. Differential 5 ' end motif was identified by Wilcoxon rank-sum test $P$ value $<0.001$ and exhibited apparent separation for ESCC against $\mathrm{HC}$ samples by unsupervised hierarchical clustering analysis (Fig. 4A). Meanwhile, NF heatmap analysis indicated that genes with differential reads coverage between promoter and background regions (Wilcoxon rank-sum test, $P$ value $<0.001$ ) held power to distinguish ESCC from HC (Fig. 4B). Additionally, our data detected that the cfDNA fragment size of ESCC was more variable and much shorter (median size $<150$ base-pairs (bp)) than that of HC group (Fig. 4C). Collectively, all three genome features of cfDNA showed promising diagnostic potential for ESCC.

As illustrated in Fig. 1 and supplementary Figure S2, healthy control individuals and patients with Early ESCC and Mid-Ad ESCC were randomly assigned into a training set (about 2/3 of samples, including 54 HC, 30 Early ESCC and 29 Mid-Ad ESCC) and a test set (about 1/3 of samples, including 17 HC, 15 Early ESCC and 19 Mid-Ad ESCC). Candidate biomarkers of the above three genomic features (5' end motif, NF and fragmentation) were screened to distinguish ESCC and HC in the training set. Least absolute shrinkage and selection operator (LASSO) regression method were applied to further reduce the number of candidate biomarkers. Eventually, 120 differential motif types, 170 differential NF genes and 10 fragment areas were selected for further analysis (supplementary Table S4A-S4C). Then, the Support Vector Machine (SVM) method was implemented for individual genomic feature-based model construction. The set of 120 motif types resulted in a discrimination model achieving an AUC value of 0.870 (95\% Cl: $0.769-0.972$ ) with sensitivity of $73.5 \%$ at specificity of $82.4 \%$ for ESCC patient classification in the test set (Fig. 4D, supplementary Figure S5A). The motif prediction model exhibited superior diagnostic power outperformed that of NF and fragmentation classifier model in cfDNA, which achieving an inferior performance with an AUC value of 0.813 (95\% Cl: $0.665-0.961)$ and 0.806 (95\% Cl: 0.677-0.936), respectively (Fig. 4D). Compared with the 5 ' end motif model, the fragmentation model demonstrated a higher sensitivity of $79.4 \%$ at the same specificity of $82.4 \%$, and NF model showed excellent sensitivity of $91.2 \%$ but a lower specificity of $70.6 \%$ (supplementary Figure S5A). Because the above three genomic features and $5 \mathrm{hmC}$ depict different aspects of the genome, we envisioned that conjoint analysis would improve diagnostic power. Using the predictive score of the four individual models as input features, an integrated diagnostic model of combining low-pass WGS cfDNA signatures and $5 \mathrm{hmC}$ biomarkers was constructed. The diagnostic power of integrated model outperformed any individual genomic features, achieving an excellent AUC value of 0.934 (95\% Cl: 0.867-1.000) with a sensitivity of $82.4 \%$, specificity of $88.2 \%$ and accuracy of $84.3 \%$ for ESCC patient classification in the test set (Fig. 4D, 4E). 
The combine scores showed an increasing trend from HC to ESCC, noting the significantly higher scores in ESCC patients with stage 0 and stage I than in subjects of $\mathrm{HC}$ in the test set $\left(P\right.$ values $=1.10 \times 10^{-2}$ and $3.31 \times 10^{-5}$, respectively, Fig. $\left.4 \mathrm{~F}\right)$, reinforcing the idea that integrated model had great potentials as a new strategy for ESCC early diagnosis and surveillance. The integrated classifier model had good but slightly reduced power to call stage III-IV patients (Fig. 4F), who in general suffered from metastasis to various tissues and are expected to have more complex tumorous DNA profiles. In addition, we compared the diagnostic performance between $5 \mathrm{hmC}$ model and integrated model on different clinical stage of ESCC samples in test set (Fig. 4G). For Early ESCC patients, especially patients with stage 0 who have lowdisease burden, the performance of integrated model was obviously superior to that of $5 \mathrm{hmC}$ model (Fig. 4G), showing evidently higher prediction accuracy (80.0\% vs 33.3\%) (supplementary Figure S5B). Integrated model and $5 \mathrm{hmC}$ model validated similar performance in stage II patients. Instead, for advanced ESCC patients (stage IIIB and IVA), 5hmC model showed an improved diagnostic performance compared to integrated model. These data demonstrated that genome-wide integration was a sensitive and robust approach which could perform better than $5 \mathrm{hmC}$-based methods for accurate diagnosis of early stage esophageal carcinoma.

\section{Discussion}

We ultimately constructed an integrated classifier using low-pass WGS and $5 \mathrm{hmC}$ biomarkers to realize our primal goal for early detection of esophageal cancer which may bring convenience to clinical practice. At first step, an investigation of the enriched $5 \mathrm{hmC}$ motifs in genome was conducted, and the outcomes showed that these existing some enriched $5 \mathrm{hmC}$ motifs in different regions like ERG, ETS1 and ETV2 which were proved to associate with cell developments, differentiation, proliferation, apoptosis and tissue remodeling, as well as GATA3, GATA4 and TRPS1 had been reported to be connected with a wide range of diseases and pathologic phenotypes, and both of these findings were consistent with our previous awareness about the biological behavior of tumors. We then compared the enriched $5 \mathrm{hmC}$ motifs among our Southern ESCC cohort (with $150 \mathrm{pb}$ paired-end sequencing mode), the Northern ESCC cohort (with 50 bp paired-end sequencing mode)[26] and colorectal cancer cohort (with 150 pb paired-end sequencing mode)[25] which suggested that some $5 \mathrm{hmC}$ motif signal changes were more conservative in ESCC patients than in other patients (such as CRC), which were not effected by different sequencing platforms. These ESCC-unique 5hmC motifs mainly consisted of up-regulated ERG, ETS1 and ETV2 of ETS family, which were reported as cancer-associated transcription factors regulating Ras-MAP kinase signaling pathways[27, 32-37]. Up-regulated EHF and ELF3 of ETS family uniquely consisted in the Northern ESCC 5hmC motifs, indicating the bias possibly due to geographic distribution or different pathological subtype compositions between these two ESCC cohorts. Other functions, such as vasculature development, muscle structure development, cell part morphogenesis/development and hippo signaling pathway were also consistent with those reported in the Northern ESCC cohort, indicating their essential roles in ESCC tumorigenesis and developments. In addition, other different functions among $5 \mathrm{hmC}$ biomarkers between our study and the Northern ESCC cohort might reveal different molecular mechanisms in different pathological subgroups of ESCC cohort or different epigenetic 
modifications resulting from different living habitats, which needed further investigation on large scale population studies.

Recently, some relevant studies showed somewhat dissatisfactory early detection efficiency which results were around $50 \%$ and $62.5 \%$ in sensitivity on esophageal cancer stage 0 and I respectively using cfDNA methylation method[38], and some of the others exhibited a good sensitivity of

93.75\% and specificity of $85.71 \%$ (AUC $=0.972$ ) in distinguishing ESCC from HC groups but slightly ignored the discussion focusing on the early stage using $5 \mathrm{hmC}$ method[26]. In the present study, using our selected $2735 \mathrm{hmC}$ marker genes, we indeed succeeded in distinguishing ESCC patients from HC individuals in both internal test set. Besides, we also made a further analysis to explore the prediction abilities of 5hmC biomarkers classifier in different stages in ESCC subgroups and found that there were significant differences between Early ESCC (stage 0 and I) and HC individuals, and the differences between stage I and $\mathrm{HC}$ samples ( $P$ value $=4.54 \times 10-3)$, stage 0 and stage I, II, III-IV were statistically significant too All the outcomes showed that the $5 \mathrm{hmC}$ indeed played an important role in tumor progress. Regretfully, in distinguishing stage 0 from $\mathrm{HC}$, we failed to work out a precise identification ( $P$ value $=0.40$, with accuracy of $33.3 \%$ ) which needed further investigation.

So far, we had verified that the $5 \mathrm{hmC}$ could definitely serve as a favorable and non-invasive tool in differential diagnosis of esophageal cancer However, with the consideration of the importance of early detection of esophageal cancer and the deficient detection efficiency between early-stage esophageal cancer (stage 0) and HC samples in our study, we aim to establish a novel diagnostic model which can achieve our goals for early detection to satisfy clinical real needs. After employing low-pass wholegenome sequencing(WGS) technology, we worked out an integrated diagnostic classifier consisting of genome-wide 5 ' end motif, nucleosome footprint (NF), fragmentation profiles and $5 \mathrm{hmC}$ biomarkers, and eventually realized an excellent AUC value of 0.934 with a sensitivity of $82.4 \%$, specificity of $88.2 \%$ and accuracy of $84.3 \%$ for ESCC patient classification in the test set, especially the scores in ESCC patients with stage 0 and 1 were significantly higher than that in $\mathrm{HC}$ subjects $\left(P\right.$ values $=1.10 \times 10^{-2}$ and $3.31 \times 10^{-}$ 5 , respectively). Briefly speaking, our findings of combination of low-pass WGS of cfDNA signatures and $5 \mathrm{hmC}$ biomarkers improved the classifier's efficiency from $65-82 \%$ of sensitivity at the specificity of $88 \%$ on an overall level. Most importantly, for stage 0 patients who had low-disease burden, the combined classifier significantly improved the prediction accuracy from 33.3-80.0\%. Though the test cohort population in this study was still limited, it was still a valuable attempt for using combined approaches to improve prediction accuracy in non-invasion early diagnosis of ESCC.

In general, more and more studies showed that integrating multi-omics detection was a promising methodology for non-invasive early diagnosis of many types of cancer. $5 \mathrm{hmC}$ was an intermediate produced through oxidation of 5-methylcytosine $(5 \mathrm{mC})$ by the oxygenase ten-eleven translocation (TET) enzyme family during $5 \mathrm{mC}$ demethylation process[39-41]. Both $5 \mathrm{mC}$ and $5 \mathrm{hmC}$ were presumed to have an important role in gene expression and regulation, and their modification changes were both observed in a wide range of malignant tumors, including ESCC[42-46]. Therefore, from a diagnostic perspective, 
combination of $5 \mathrm{mC}$ and $5 \mathrm{hmC}$ signal detection, as well as WGS features of cfDNA in this study to construct classifiers to further improve the specificity and sensitivity for early diagnosis of different subtypes and stages of ESCC patients was worthy of attempts. Here, an integrated model was worked out to realize early detection for esophageal cancer in a non-invasive approach that could be conveniently applied to clinical practices. Of course, further investigations about the stability of this model, the discriminating capabilities for different subtypes of esophageal cancer, or the practical application values are needed to execute. We sincerely hope that this study could provide an innovative clinical diagnostic strategy and could be put into practical use in the future which ultimately bring our patients with positive benefits.

\section{Conclusions}

This study suggests that the blood-based $5 \mathrm{hmC}$ integrated with low-pass WGS model could improve the accurate diagnosis of early stage ESCC, particularly for diagnosis of very early stage ESCC.

\section{Methods}

\section{Study participants and clinical features}

All the patients selected to experimental group were diagnosed with esophageal squamous cell carcinoma (ESCC) and were confirmed cytopathologically and histologically, also, the patients were restricted to which on initial treatments from 0-PIV diagnosed by using the esophagus and esophagogastric junction of the eighth edition of the AJCC/UICC cancer staging manuals[47]. Except for living habits (especially hot food preference) and family disease history, other hazard covariates like BMI, smoking and drinking were kept consistent to the greatest extent. For patients subjected to control group, participants were selected from health examine center of our hospital, and no esophageal squamous cell carcinoma and other relevant diseases were observed. The other standards were consistent with experimental group. We excluded patients who used to receive surgery, chemoradiotherapy or immunotherapy, and patients who suffered from other illnesses like leukemia, neurodegenerative disease or other tumoral disorders. In total, 150 participants consisting of 100 esophageal squamous cell carcinoma (ESCC) patients and 50 healthy controls (HC) were enrolled from Nanfang Hospital of Southern Medical University in March 2019 to December 2020 and named as a Southern ESCC cohort since the patients were mainly from the Southern of China. Moreover, we added additional 21 healthy control samples used in a previously published study[25] to our HC cohort.

\section{Blood Sample Preparation And Cfdna Extraction}

Peripheral blood specimens $(10 \mathrm{~mL} /$ subject) were obtained from patients who were newly diagnosed and had never received any medication or radical treatment for disease, which was prior to any biopsy or surgical resection. $71 \mathrm{HC}$ blood samples were collected at the time of visiting the clinic for routine 
physical examination. All peripheral blood samples were stored in cell-free tubes (Streck, USA) at $4^{\circ} \mathrm{C}$ for no more than 72 hours before being separated into plasma and stored at $-80^{\circ} \mathrm{C}$ in the laboratory. The plasma cell free DNA (cfDNA) was isolated using the MagMAX Cell-Free DNA Isolation Kit (Thermo, USA) according to the manufacturer's protocol. The quality of purified DNA was quantified by Qubit® 4.0 Fluorometer (Life Technologies, USA), and the DNA fragment size composition was assayed by Fragment Analyzer (Agilent, USA).

\section{5hmc Sequencing And Data Processing $5 \mathrm{hmC}$ library construction and sequencing}

$5 \mathrm{hmC}$ library construction was performed according to the method previously described[23]. Briefly, the purified cfDNA (5-20 ng) were end-repaired and A tailed (5X ER/A-Tailing Enzyme Mix, Enzymatics, USA) then ligated with T-adaptors on both ends (WGS Ligase, Enzymatics, USA), to result in pre-library. Subsequently, ligated DNA was incubated in a $25 \mu$ l solution containing $50 \mathrm{mM} \mathrm{HEPES}$ buffer $(\mathrm{pH}=8.0)$, $25 \mathrm{mM}$ MgCl2, $60 \mu \mathrm{M}$ UDP-6-N3-Glc (Active Motif, USA) and $12.5 \mathrm{U} \beta \mathrm{GT}$ (Thermo, USA) for $2 \mathrm{~h}$ at $37^{\circ} \mathrm{C}$. Then, $2.5 \mu$ I DBCO-PEG4-biotin (Click Chemistry Tools, USA) was added to the reaction mixture and incubated for $2 \mathrm{~h}$ at $37^{\circ} \mathrm{C}$. The purified DNA was incubated with $0.5 \mu \mathrm{l}$ M270 Streptavidin beads (Life Technologies, USA) pre-blocked with salmon sperm DNA in buffer 1 ( $5 \mathrm{mM}$ Tris pH 7.5, 0.5 mM EDTA, $1 \mathrm{M}$ $\mathrm{NaCl}$ and $0.2 \%$ Tween 20) for $30 \mathrm{~min}$. Afterwards, DNA fragments containing $5 \mathrm{hmC}$ features were subjected to PCR amplification, followed by the purification of the PCR products using AMPure XP beads according to the manufacturer's instructions. Finally, sequenced using Illumina NovaSeq 6000 platform.

\section{Mapping And Sequencing Quality Control}

The raw sequencing reads were removed adaptor and end sequence by trim_galore software (https: //www.bioinformatics. babraham. ac.uk/projects/trim_galore/)[48]. Acquired clean data were aligned to the human reference genome (hg19/GRCh37) by Bowtie2 v2.2.5 (http://bowtiebio.sourceforge.net/bowtie2/index.shtml)[49]. Picard Tools (http://broadinstitute.github.io/picard/) and SAMtools (http://samtools.sourceforge.net/)[50] were used to process and filter PCR duplicates for mapped BAM files. Reads with duplicate ratio less than $65 \%$ and enrichment efficiency over 95 -fold passed the quality control and were used for further analysis.

\section{5hmc Peak Identification}

Model-based analysis of ChIP-seq[51] was used to identify the $5 \mathrm{hmC}$-enriched regions in each sample (the q value cut-off to call significant regions is 0.01 ; model fold $=[5,50]$ ). The peaks with high enrichment and significance ( $\mathrm{q}<1 \mathrm{E}-12$; fold enrichment $>8$ ) in all samples were considered as highly reliable $5 \mathrm{hmC}$-enriched peaks. The $5 \mathrm{hmC}$ enrichment level was expressed as fragments per kilobase of 
5hmC-DNA per million fragments mapped (FPKM). The genomic annotation of $5 \mathrm{hmC}$ peak regions was performed using annotatr[52] and the genome-wide distribution of $5 \mathrm{hmC}$ was visualized using the Integrated Genomics Viewer[53, 54]. The metagene profile was generated using ngsplot[55].

\section{Differential 5hmc Peak Regions Detection}

The differential $5 \mathrm{hmC}$ peak regions between healthy control group and ESCC group were identified using DESeq2 package[56] with $P$ value $<0.01$. De novo motif analysis around differential $5 \mathrm{hmC}$ peaks was performed using HOMER software (version 4.9). Functional gene ontology (GO) and Kyoto Encyclopedia of Genes and Genomes (KEGG) pathway analyses were performed using an online tool of metascape (http://metascape.org/)[57].

\section{5hmc Biomarkers Identification And Evaluating Performance}

$5 \mathrm{hmC}$ candidate biomarkers for cancer prediction models were identified firstly based on Wilcoxon ranksum test $(P$ values $<0.001)$ between ESCC and HC groups and then processed to reduce the number of $5 \mathrm{hmC}$ biomarkers based on Recursive Feature Elimination - Cross Validation (RFECV) approach in the training set ( $54 \mathrm{HC}, 34$ Early ESCC and 31 Mid-Ad ESCC). The remaining samples in each group were used as the internal test set (including 17 HC, 16 Early ESCC and 19 Mid-Ad ESCC). In addition, we downloaded 150 esophageal cancer plasma-5hmC data and 183 healthy control plasma-5hmC data from the article previously published (nominated as the Northern ESCC cohort)[15] as an external test set to validate our results.

The selected 273 differential $5 \mathrm{hmC}$ biomarkers using for sample identification to be ESCC or $\mathrm{HC}$ were analyzed by the principal component analysis (PCA). A heatmap of R package (https://cran.rproject.org/web/packages/pheatmap/ index.html) was used to visualize hierarchical clustering and the distance in a heatmap figure[58]. $5 \mathrm{hmC}$ biomarkers were further processed for classification model construction based on a supervised two-class Support Vector Machine (SVM) method. To optimize the classification model and ensure the significance of each potential $5 \mathrm{hmC}$ biomarkers, the GridSearchCV package in Python in conjunction with cross-validation were performed to obtain the optimal parameters for SVM based on the Gaussian kernel (kernel='rbf', gamma $=0.001$, the penalty parameter $C=4$ ). The defined 5hmC-DNA regions and their corresponding genes were finally applied to classify the test set samples.

\section{Low-pass Whole Genome Sequencing And Data Processing WGS library construction and sequencing}


1-10 ng cfDNA were end-repaired and A-tailed (Berry, China) then ligated with T-adaptors (Berry, China), to result in pre-library. The pre-libraries were purified by Clean NGS beads (VdoBiotech, China), followed by being quantified by the KAPA Library Quantification Kit (Kapa Biosystems, USA). cfDNA fragment size was confirmed using Bioanalyzer (Agilent, USA). Sequencing libraries were pooled at equal amount and then sequenced on an Illumina CN500 platform (Illumina, San Diego, USA) with an average coverage of $2 x$.

\section{Mapping And Sequencing Quality Control}

The raw sequencing reads were removed adaptor and end sequence by fastp software (https://github.com/OpenGene/fastp). Acquired clean data were aligned to human reference genome (hg19/GRCh37) using bwa-mem (https://github.com/lh3/bwa). Duplicate reads were marked by sambamba (https://github.com/biod/sambamba/). Sequencing data were further processed by SAMtools (http://samtools.sourceforge.net/)[50] to get rid of marked duplicates, unmapped reads and low quality reads. Reads with duplicate rate less than $15 \%$ and mapping rate more than $95 \%$ passed the quality control and were used for further analysis.

\section{Wgs-based Biomarkers Identification And Integrated Model Construction}

To select more effective biomarkers for distinguishing ESCC samples from healthy controls, all samples were randomly separated into two subsets: the training set consisted of $54 \mathrm{HC}, 30$ Early ESCC and 29 Mid-Ad ESCC, and the test set consisted of the remaining samples (including $17 \mathrm{HC}, 15$ Early ESCC and 19 Mid-Ad ESCC). The Wilcoxon rank-sum test was used to compare biomarker features between ESCC and HC groups. Least Absolute Shrinkage and Selection Operator (LASSO) methods were applied to further reduce the number of biomarkers in the training set. The detailed selecting process was performed as follows.

$5^{\prime}$ end Motif: 256 different types of 4 mer 5 ' end motif were identified and calculated their percentages (using pysam (https://pysam.readthedocs.io/en/latest/)) without considering chromosome $Y$ and unidentifiable bases. Following motif types were filtered out: 1) $P \geq 0.05$ in Wilcoxon rank-sum test between ESCC and HC groups; 2) weight of 0 via LASSO. Eventually, 120 motif types were left for further analysis.

\section{Nucleosome footprint (NF)}

We obtained all transcripts of coding genes, microRNAs and long non-coding RNAs (LncRNAs) and calculated the distance between transcripts. The transcripts with the distance more than $200 \mathrm{bp}$ were retained. If the distance between two transcripts less than $200 \mathrm{bp}$, the longer transcript was retained. A total of 57151 transcripts of 30588 genes were recruited for analysis. The promoter region and 
background region of transcripts were divided, and the reads number of different regions was counted with featureCounts[59]. NF score of each gene is calculated as

NF Score= $($ background1 + background2) $/ 2$-Promotor

Following genes were filtered out: 1) more than $10 \%$ of the total samples showing an NF score of 0 ;2) $P$ $\geq 0.001$ in Wilcoxon rank-sum test between ESCC and HC groups; 3 ) weight of 0 via LASSO. Eventually, 170 genes were left for further analysis.

Fragment: The whole genome except $Y$ chromosome was divided into $1 \mathrm{M}$ sized bins, resulting in 3055 areas. Pysam (https://pysam.readthedocs.io/en/latest/) was used to calculate the length of insertion fragment and ratio of short/long fragment in different regions. LASSO was then used to filter out areas with a weight of 0 , and finally 10 areas were retained.

Thereafter, the Support Vector Machine (SVM) method was implemented for individual genomic featurebased model construction. 10-fold cross-validation method was employed to optimize the combination of the parameters in the training set, and cut-off value was set at the point with the best diagnostic accuracy. To obtain the best diagnostic model, logistic regression model was generated using the predictive score of the four individual models as input features to integrate the outcome of each model based on the training dataset. The logistic Score was calculated as follows.

Logistic Score $=\exp (Z) /(1+\exp (Z))$, where $Z=-2.57+(3.35 \times N F)+\llbracket 0.05 \times$ Fragment $\rrbracket+\llbracket 0.75 \times$ Motif $\rrbracket+\rrbracket 1.74 \times 5 \mathrm{hmC} \square$

Receiver operating characteristic (ROC) curves[60] were generated to evaluate the performance of a prediction algorithm, using the pROC[61] library in the R package. Sensitivity and specificity were estimated at the score cut-off that maximizes the sum of sensitivity and specificity using the ROCR library in the R package.

\section{Statistics}

The statistical methods used were stated in the above methods. The R code related to classifier detection and modelling is available upon request.

\section{Declarations}

\section{Ethics approval and consent to participate}

The study was approved by the ethics committee of the Nanfang Hospital, Southern Medical University, Guangzhou, China (reference: NFEC-2019-014) and was also registered with ClinicalTrials.gov (reference: NCT03922230). Besides, this study was conducted by the approval of the Institutional Review Board of 
Nanfang Hospital of Southern Medical University, and the written informed consents were obtained from all participants according to the institutional guidelines.

\section{Consent for publication}

All the authors agreed to submit and publish the manuscript to Journal of Hematology \& Oncology.

\section{Data availability statement}

All of the raw and processed data used in this study have been uploaded to the Genome Sequence Archive depository (https://ngdc.cncb.ac.cn/gsa)[62] with the Accession Number (HRA001476).

Conflict of interest:SW, HL, FS, SW and XZ are employees of Berry Oncology Corporation.Other authors had no declaration of conflicts of interests.

Funding. Science and Technology Planning Project of Guangdong Province(2017B020226005).

\section{Author contributions}

$\mathrm{KC}$, SW and DLdesigned the research. DL,XW, JZ and XD recruited the examinees. DL, XW and SF got consents and collected raw data. DL, XY, HL and SWwrote the manuscript. SW, XZ,FS conducted bioinformatics analysisanddata visualization. All authors reviewed and approved the manuscript.

\section{Acknowledgments}

Thanks for the help of Professor Side Liu, Dr. Jianqun Cai and Dr. Jing Wang from Department of Gastroenterology, Ms. Li Zhen from Department of General Surgery, Ms. Yu Guo from Department of Huiqiao Building, and Ms. Mei Li from Department of Thoracic Surgery, Nanfang Hospital, Southern Medical University, Guangzhou, China. This study was funded by the Science and Technology Planning Project of Guangdong Province (2017B020226005).

\section{References}

1. Sung H, Ferlay J, Siegel RL, Laversanne M, Soerjomataram I, Jemal A, Bray F: Global Cancer Statistics 2020: GLOBOCAN Estimates of Incidence and Mortality Worldwide for 36 Cancers in 185 Countries. CA: a cancer journal for clinicians 2021, 71(3):209-249.

2. Smyth EC, Lagergren J, Fitzgerald RC, Lordick F, Shah MA, Lagergren P, Cunningham D: Oesophageal cancer. Nature reviews Disease primers 2017, 3:17048.

3. Allemani C, Matsuda T, Di Carlo V, Harewood R, Matz M, Nikšić M, Bonaventure A, Valkov M, Johnson CJ, Estève $J$ et al: Global surveillance of trends in cancer survival 2000-14 (CONCORD-3): analysis of individual records for 37513025 patients diagnosed with one of 18 cancers from 322 populationbased registries in 71 countries. Lancet 2018, 391(10125):1023-1075. 
4. Wani S, Yadlapati R, Singh S, Sawas T, Katzka DA, Hall M, Bergman J, Canto MI, Chak A, Corley DA et al: Post-Endoscopy Esophageal Neoplasia in Barrett's Esophagus: Consensus Statements from an International Expert Panel. Gastroenterology 2021.

5. di Pietro M, Canto MI, Fitzgerald RC: Endoscopic Management of Early Adenocarcinoma and Squamous Cell Carcinoma of the Esophagus: Screening, Diagnosis, and Therapy. Gastroenterology 2018, 154(2):421-436.

6. Wan JCM, Massie C, Garcia-Corbacho J, Mouliere F, Brenton JD, Caldas C, Pacey S, Baird R, Rosenfeld N: Liquid biopsies come of age: towards implementation of circulating tumour DNA. Nature reviews Cancer 2017, 17(4):223-238.

7. Thierry AR, El Messaoudi S, Gahan PB, Anker P, Stroun M: Origins, structures, and functions of circulating DNA in oncology. Cancer metastasis reviews 2016, 35(3):347-376.

8. Cristiano S, Leal A, Phallen J, Fiksel J, Adleff V, Bruhm DC, Jensen S $\varnothing$, Medina JE, Hruban C, White JR et al: Genome-wide cell-free DNA fragmentation in patients with cancer. Nature 2019, 570(7761):385-389.

9. Luo H, Wei W, Ye Z, Zheng J, Xu RH: Liquid Biopsy of Methylation Biomarkers in Cell-Free DNA. Trends in molecular medicine 2021, 27(5):482-500.

10. Jones PA: Functions of DNA methylation: islands, start sites, gene bodies and beyond. Nature reviews Genetics 2012, 13(7):484-492.

11. Baylin SB, Jones PA: A decade of exploring the cancer epigenome - biological and translational implications. Nature reviews Cancer 2011, 11(10):726-734.

12. Dor Y, Cedar H: Principles of DNA methylation and their implications for biology and medicine. The Lancet 2018, 392(10149):777-786.

13. Branco MR, Ficz G, Reik W: Uncovering the role of 5-hydroxymethylcytosine in the epigenome. Nature reviews Genetics 2011, 13(1):7-13.

14. Guler GD, Ning Y, Ku CJ, Phillips T, McCarthy E, Ellison CK, Bergamaschi A, Collin F, Lloyd P, Scott A et al: Detection of early stage pancreatic cancer using 5-hydroxymethylcytosine signatures in circulating cell free DNA. Nature communications 2020, 11(1):5270.

15. Zhang J, Han X, Gao C, Xing Y, Qi Z, Liu R, Wang Y, Zhang X, Yang YG, Li X et al: 5-Hydroxymethylome in Circulating Cell-free DNA as A Potential Biomarker for Non-small-cell Lung Cancer. Genomics, proteomics \& bioinformatics 2018, 16(3):187-199.

16. Gilat N, Tabachnik T, Shwartz A, Shahal T, Torchinsky D, Michaeli Y, Nifker G, Zirkin S, Ebenstein Y: Single-molecule quantification of 5-hydroxymethylcytosine for diagnosis of blood and colon cancers. Clinical epigenetics 2017, 9:70.

17. Li W, Zhang X, Lu X, You L, Song Y, Luo Z, Zhang J, Nie J, Zheng W, Xu D et al: 5Hydroxymethylcytosine signatures in circulating cell-free DNA as diagnostic biomarkers for human cancers. Cell research 2017, 27(10):1243-1257.

18. Song CX, Yin S, Ma L, Wheeler A, Chen Y, Zhang Y, Liu B, Xiong J, Zhang W, Hu J et al: 5Hydroxymethylcytosine signatures in cell-free DNA provide information about tumor types and 
stages. Cell research 2017, 27(10):1231-1242.

19. Cai J, Chen L, Zhang Z, Zhang X, Lu X, Liu W, Shi G, Ge Y, Gao P, Yang Y et al: Genome-wide mapping of 5-hydroxymethylcytosines in circulating cell-free DNA as a non-invasive approach for early detection of hepatocellular carcinoma. Gut 2019, 68(12):2195-2205.

20. Zviran A, Schulman RC, Shah M, Hill STK, Deochand S, Khamnei CC, Maloney D, Patel K, Liao W, Widman AJ et al: Genome-wide cell-free DNA mutational integration enables ultra-sensitive cancer monitoring. Nat Med 2020, 26(7):1114-1124.

21. Chabon JJ, Hamilton EG, Kurtz DM, Esfahani MS, Moding EJ, Stehr H, Schroers-Martin J, Nabet BY, Chen $B$, Chaudhuri AA et al: Integrating genomic features for non-invasive early lung cancer detection. Nature 2020, 580(7802):245-251.

22. Mathios D, Johansen JS, Cristiano S, Medina JE, Phallen J, Larsen KR, Bruhm DC, Niknafs N, Ferreira $\mathrm{L}$, Adleff $\mathrm{V}$ et al: Detection and characterization of lung cancer using cell-free DNA fragmentomes. Nature communications 2021, 12(1):5060.

23. Chen L, Abou-Alfa GK, Zheng B, Liu JF, Bai J, Du LT, Qian YS, Fan R, Liu XL, Wu L et al: Genome-scale profiling of circulating cell-free DNA signatures for early detection of hepatocellular carcinoma in cirrhotic patients. Cell research 2021, 31(5):589-592.

24. Szymanski JJ, Sundby RT, Jones PA, Srihari D, Earland N, Harris PK, Feng W, Qaium F, Lei H, Roberts $D$ et al: Cell-free DNA ultra-low-pass whole genome sequencing to distinguish malignant peripheral nerve sheath tumor (MPNST) from its benign precursor lesion: A cross-sectional study. PLoS Med 2021, 18(8):e1003734.

25. Xiao Z, Wu W, Wu C, Li M, Sun F, Zheng L, Liu G, Li X, Yun Z, Tang J et al: 5-Hydroxymethylcytosine signature in circulating cell-free DNA as a potential diagnostic factor for early-stage colorectal cancer and precancerous adenoma. Molecular oncology 2021, 15(1):138-150.

26. Tian X, Sun B, Chen C, Gao C, Zhang J, Lu X, Wang L, Li X, Xing Y, Liu R et al: Circulating tumor DNA 5-hydroxymethylcytosine as a novel diagnostic biomarker for esophageal cancer. Cell research 2018, 28(5):597-600.

27. Oikawa T: ETS transcription factors: possible targets for cancer therapy. Cancer science 2004, 95(8):626-633.

28. Romano $O$, Miccio A: GATA factor transcriptional activity: Insights from genome-wide binding profiles. IUBMB Life 2020, 72(1):10-26.

29. Chen D, Wang K, Li X, Jiang M, Ni L, Xu B, Chu Y, Wang W, Wang H, Kang H et al: FOXK1 plays an oncogenic role in the development of esophageal cancer. Biochem Biophys Res Commun 2017, 494(1-2):88-94.

30. Serpas L, Chan RWY, Jiang P, Ni M, Sun K, Rashidfarrokhi A, Soni C, Sisirak V, Lee W-S, Cheng SH et al: deletion causes aberrations in length and end-motif frequencies in plasma DNA. Proc Natl Acad Sci U S A 2019, 116(2):641-649.

31. Zhao Y, Wang J, Liang F, Liu Y, Wang Q, Zhang H, Jiang M, Zhang Z, Zhao W, Bao Y et al: NucMap: a database of genome-wide nucleosome positioning map across species. Nucleic Acids Res 2019, 
47(D1):D163-D169.

32. Wei GH, Badis G, Berger MF, Kivioja T, Palin K, Enge M, Bonke M, Jolma A, Varjosalo M, Gehrke AR et al: Genome-wide analysis of ETS-family DNA-binding in vitro and in vivo. The EMBO journal 2010 , 29(13):2147-2160.

33. Dittmer J: The role of the transcription factor Ets1 in carcinoma. Seminars in cancer biology 2015, 35:20-38.

34. Saeki H, Kuwano H, Kawaguchi H, Ohno S, Sugimachi K: Expression of ets- 1 transcription factor is correlated with penetrating tumor progression in patients with squamous cell carcinoma of the esophagus. Cancer 2000, 89(8):1670-1676.

35. Saeki H, Oda S, Kawaguchi H, Ohno S, Kuwano H, Maehara Y, Sugimachi K: Concurrent overexpression of Ets-1 and c-Met correlates with a phenotype of high cellular motility in human esophageal cancer. Int J Cancer 2002, 98(1).

36. Baltrunaite K, Craig MP, Palencia Desai S, Chaturvedi P, Pandey RN, Hegde RS, Sumanas S: ETS transcription factors Etv2 and Fli1b are required for tumor angiogenesis. Angiogenesis 2017, 20(3):307-323.

37. Adamo P, Ladomery MR: The oncogene ERG: a key factor in prostate cancer. Oncogene 2016, 35(4):403-414.

38. Qiao G, Zhuang W, Dong B, Li C, Xu J, Wang G, Xie L, Zhou Z, Tian D, Chen G et al: Discovery and validation of methylation signatures in circulating cell-free DNA for early detection of esophageal cancer: a case-control study. BMC medicine 2021, 19(1):243.

39. Gilat N, Tabachnik T, Shwartz A, Shahal T, Torchinsky D, Michaeli Y, Nifker G, Zirkin S, Ebenstein Y: Single-molecule quantification of 5-hydroxymethylcytosine for diagnosis of blood and colon cancers. Clinical epigenetics 2017, 9:70.

40. Song C-X, Yin S, Ma L, Wheeler A, Chen Y, Zhang Y, Liu B, Xiong J, Zhang W, Hu J et al: 5Hydroxymethylcytosine signatures in cell-free DNA provide information about tumor types and stages. Cell research 2017, 27(10):1231-1242.

41. Li W, Zhang X, Lu X, You L, Song Y, Luo Z, Zhang J, Nie J, Zheng W, Xu D et al: 5Hydroxymethylcytosine signatures in circulating cell-free DNA as diagnostic biomarkers for human cancers. Cell research 2017, 27(10):1243-1257.

42. Shi X, Yu Y, Luo M, Zhang Z, Shi S, Feng X, Chen Z, He J: Loss of 5-Hydroxymethylcytosine Is an Independent Unfavorable Prognostic Factor for Esophageal Squamous Cell Carcinoma. PloS one 2016, 11(4):e0153100.

43. Murata A, Baba Y, Ishimoto T, Miyake K, Kosumi K, Harada K, Kurashige J, Iwagami S, Sakamoto Y, Miyamoto $Y$ et al: TET family proteins and 5-hydroxymethylcytosine in esophageal squamous cell carcinoma. Oncotarget 2015, 6(27):23372-23382.

44. Li D, Zhang L, Liu Y, Sun H, Onwuka JU, Zhao Z, Tian W, Xu J, Zhao Y, Xu H: Specific DNA methylation markers in the diagnosis and prognosis of esophageal cancer. Aging (Albany NY) 2019, 11(23):11640-11658. 
45. Su J, Wu G, Ye Y, Zhang J, Zeng L, Huang X, Zheng Y, Bai R, Zhuang L, Li M et al: NSUN2-mediated RNA 5-methylcytosine promotes esophageal squamous cell carcinoma progression via LIN28Bdependent GRB2 mRNA stabilization. Oncogene 2021, 40(39):5814-5828.

46. Kit OI, Vodolazhskiy DI, Kolesnikov EN, Timoshkina NN: [Epigenetic markers of esophageal cancer: DNA methylation]. Biomed Khim 2016, 62(5):520-526.

47. Rice TW, Ishwaran H, Ferguson MK, Blackstone EH, Goldstraw P: Cancer of the Esophagus and Esophagogastric Junction: An Eighth Edition Staging Primer. J Thorac Onco/2017, 12(1):36-42.

48. Lindgreen S: AdapterRemoval: easy cleaning of next-generation sequencing reads. BMC Res Notes 2012, 5:337.

49. Langmead B, Salzberg SL: Fast gapped-read alignment with Bowtie 2. Nat Methods 2012, 9(4):357359.

50. Etherington GJ, Ramirez-Gonzalez RH, MacLean D: bio-samtools 2: a package for analysis and visualization of sequence and alignment data with SAMtools in Ruby. Bioinformatics 2015, 31(15):2565-2567.

51. Grytten I, Rand KD, Nederbragt AJ, Storvik GO, Glad IK, Sandve GK: Graph Peak Caller: Calling ChIPseq peaks on graph-based reference genomes. PLoS Comput Biol 2019, 15(2):e1006731.

52. Cavalcante RG, Sartor MA: annotatr: genomic regions in context. Bioinformatics 2017, 33(15):23812383.

53. Thorvaldsdóttir H, Robinson JT, Mesirov JP: Integrative Genomics Viewer (IGV): high-performance genomics data visualization and exploration. Brief Bioinform 2013, 14(2):178-192.

54. Robinson JT, Thorvaldsdóttir H, Winckler W, Guttman M, Lander ES, Getz G, Mesirov JP: Integrative genomics viewer. Nat Biotechnol 2011, 29(1):24-26.

55. Shen L, Shao N, Liu X, Nestler E: ngs.plot: Quick mining and visualization of next-generation sequencing data by integrating genomic databases. BMC Genomics 2014, 15:284.

56. Love MI, Huber W, Anders S: Moderated estimation of fold change and dispersion for RNA-seq data with DESeq2. Genome Biol 2014, 15(12):550.

57. Tripathi S, Pohl MO, Zhou Y, Rodriguez-Frandsen A, Wang G, Stein DA, Moulton HM, DeJesus P, Che J, Mulder LCF et al: Meta- and Orthogonal Integration of Influenza "OMICs" Data Defines a Role for UBR4 in Virus Budding. Cell Host Microbe 2015, 18(6):723-735.

58. Galili T, O'Callaghan A, Sidi J, Sievert C: heatmaply: an R package for creating interactive cluster heatmaps for online publishing. Bioinformatics 2018, 34(9):1600-1602.

59. Liao Y, Smyth GK, Shi W: featureCounts: an efficient general purpose program for assigning sequence reads to genomic features. Bioinformatics 2014, 30(7):923-930.

60. Sing T, Sander O, Beerenwinkel N, Lengauer T: ROCR: visualizing classifier performance in $\mathbf{R}$. Bioinformatics 2005, 21(20):3940-3941.

61. Robin X, Turck N, Hainard A, Tiberti N, Lisacek F, Sanchez J-C, Müller M: pROC: an open-source package for $\mathrm{R}$ and $\mathrm{S}+$ to analyze and compare ROC curves. BMC Bioinformatics 2011, 12:77. 
62. Wang Y, Song F, Zhu J, Zhang S, Yang Y, Chen T, Tang B, Dong L, Ding N, Zhang Q et al: GSA: Genome Sequence Archive<sup/>. Genomics, proteomics \& bioinformatics 2017, 15(1):14-18.

\section{Figures}

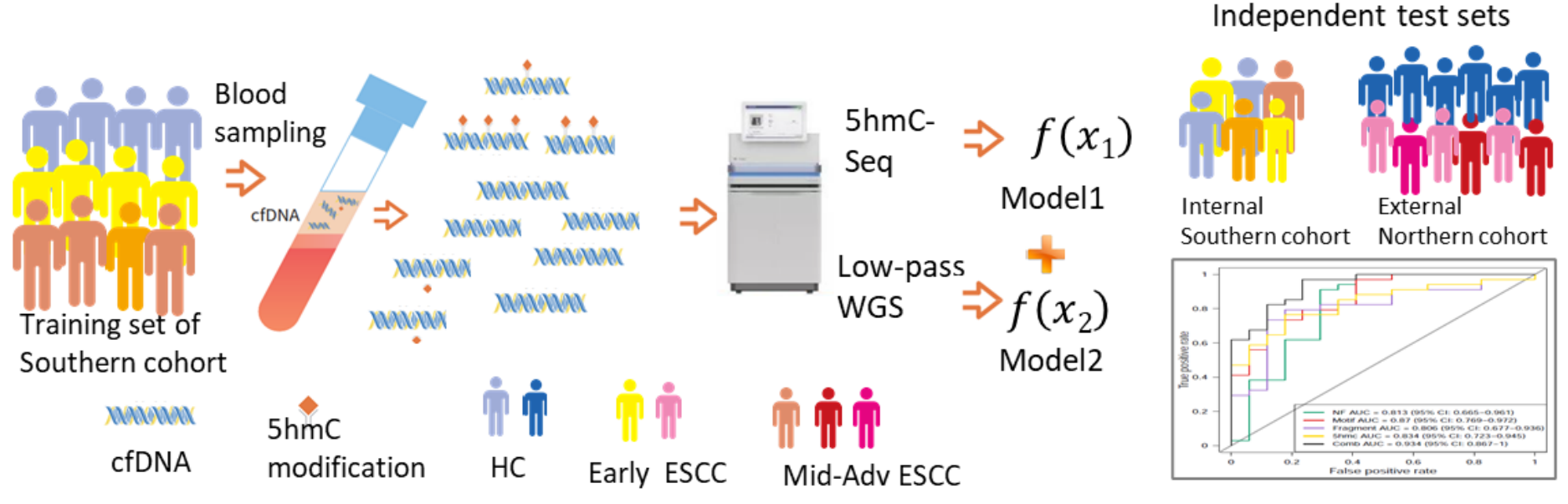

Figure 1

Sketch map of study design and research pipeline for early detection of ESCC.

5hmC-based diagnostic model and low-pass WGS-based diagnostic model were developed for identifying ctDNA from plasma cfDNA using machine learning approach.Totally 171 subjects were involved as a Southern ESCC cohort and blood samples were collected to perform $5 \mathrm{hmC}$-seqeuncing and low-pass WGS, respectively. Two-thirds subjects were randomly selected as a training set and the left one-third subjects were used as an independently internal Southern-ESCC test set to evaluate the model performance. A downloaded ESCC-5hmC dataset was used as an independently external Northern-ESCC test set. Detail research pipeline was illustrated in supplementary Figure S2.

cfDNA, cell-free tumor DNA. cfDNA, cell-free DNA; HC, healthy controls individuals; ESCC, esophageal squamous cell carcinoma; Mid-Ad, middle-advanced; $5 \mathrm{hmC}$, 5-hydroxymethylcytosines; WGS, whole genome sequencing. 

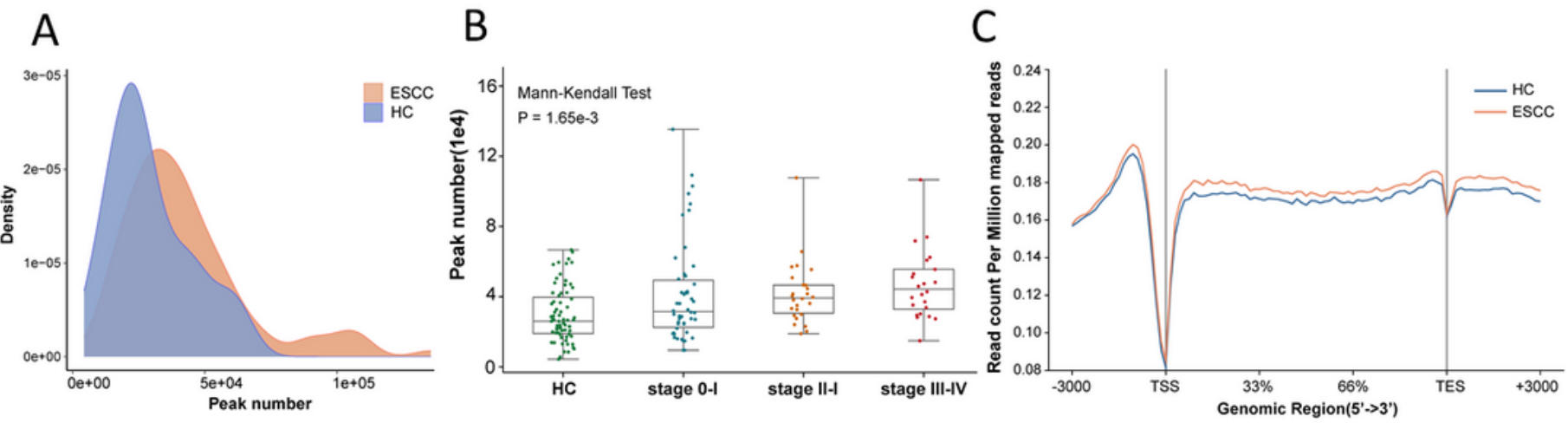

D
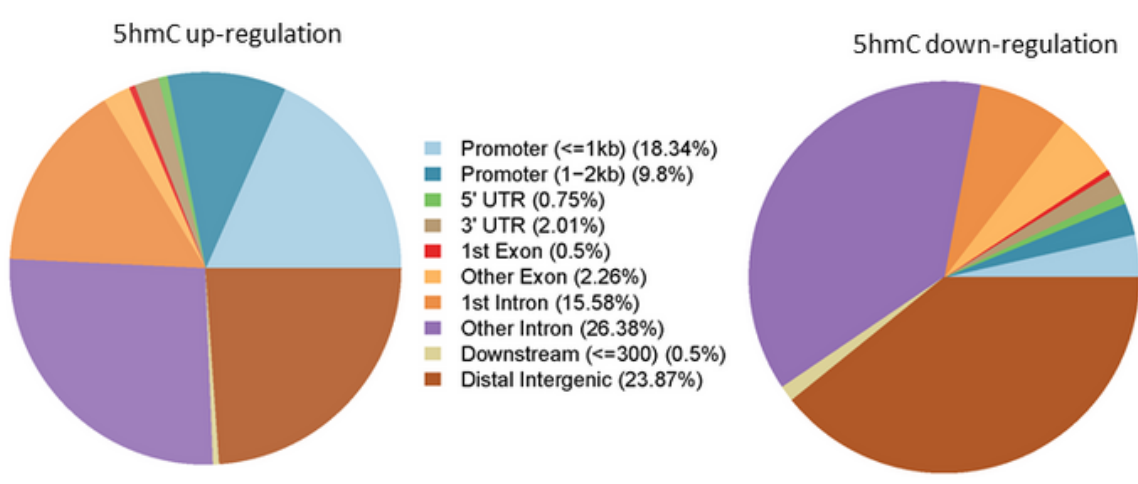

In Promoter $(<=1 \mathrm{~kb})(3.52 \%)$

E Promoter (1-2kb) (2.64\%)

= $5^{\prime}$ UTR $(0.88 \%)$

= $3^{\prime}$ UTR $(1.76 \%)$

- 1st Exon (0.44\%)

ather Exon $(5.29 \%)$

=1st Intron $(7.49 \%)$

- Other Intron $(37.44 \%)$

Downstream $(<=300)(1.32 \%)$

n Distal Intergenic (39.21\%)

E $5 \mathrm{hmC}$ up-regulation

$5 \mathrm{hmC}$ down-regulation

\section{ACAGGAATC

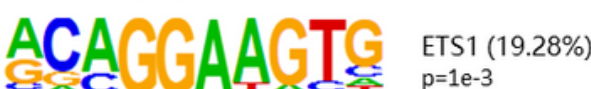

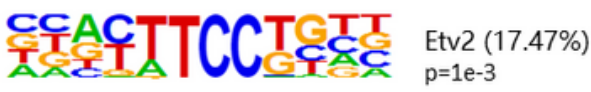

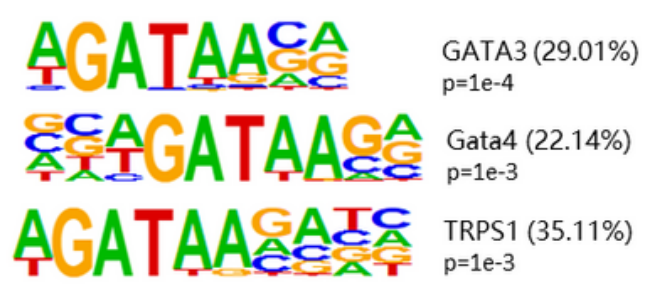

F

$5 \mathrm{hmC}$ up-regulation

$5 \mathrm{hmC}$ down-regulation
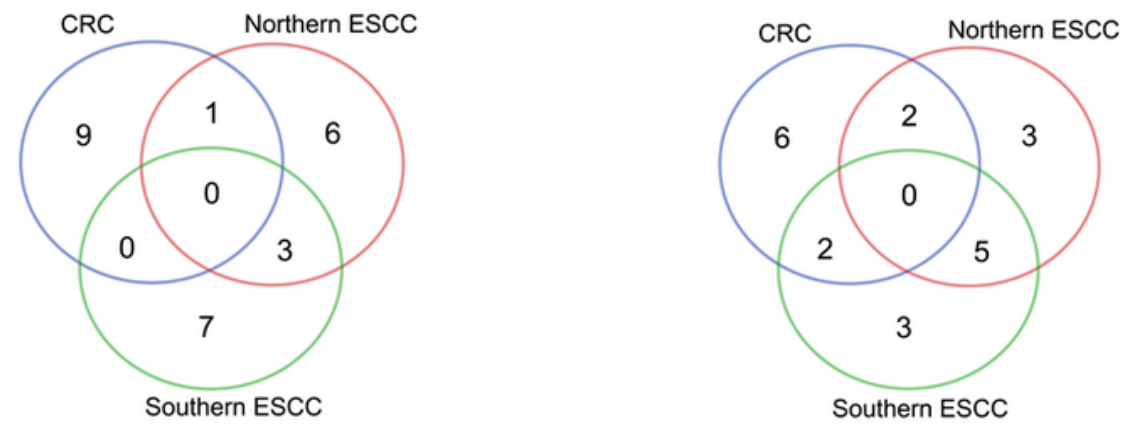

Figure 2

Genome-wide distribution of 5hmC signals in plasma cfDNA of ESCC and HC individuals.

(A) Comparison of density distribution of $5 \mathrm{hmC}$ peaks number in plasma samples from $71 \mathrm{HC}$ and 100 patients with ESCC. 
(B) Comparison of the total number of $5 \mathrm{hmC}$ peaks in $\mathrm{HC}$ and ESCC patients with stage 0 -I, II, III-IV. Each dot depicts an individual cfDNA sample. $P$ value shows statistical significance by Mann-Kendall Test.

(C) Metagene profiles of mean values of $5 \mathrm{hmC}$ read counts on the regions from TSS to TES with the flanking 3000-bp in HC and ESCC samples.

(D) Distribution of differential $5 \mathrm{hmC}$ peaks in genomic elements in ESCC samples versus $\mathrm{HC}$ samples.

(E) Top enriched known transcription factor binding motifs detected in differential $5 \mathrm{hmC}$ peaks (left: $5 \mathrm{hmC}$ up-regulation; right: $5 \mathrm{hmC}$ down-regulation). Motif information was obtained from the Homer motif database. The value in parenthesis represents the percentage of target sequences enriched with the binding motif of the indicated transcription factor.

(F) Venn plot of the top 10-significantly enrichment $5 \mathrm{hmC}$ motifs in our Southern ESCC group, the Northern ESCC group and the CRC group (left: $5 \mathrm{hmC}$ up-regulation; right: $5 \mathrm{hmC}$ down-regulation).

$\mathrm{HC}$, healthy controls; ESCC, esophageal squamous cell carcinoma; CRC, colorectal cancer; TSS, transcription start sites; TES, transcription end site; $5 \mathrm{hmC}$, 5-hydroxymethylcytosines. 

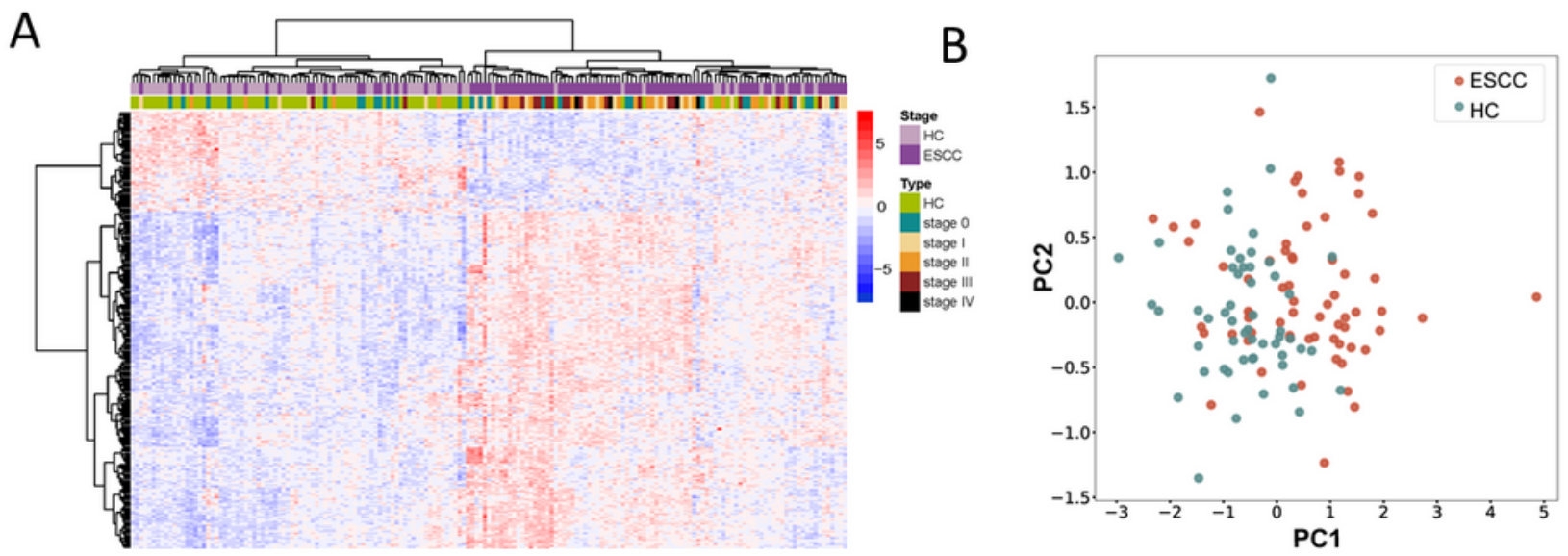

\section{C $\mathrm{GO}$}

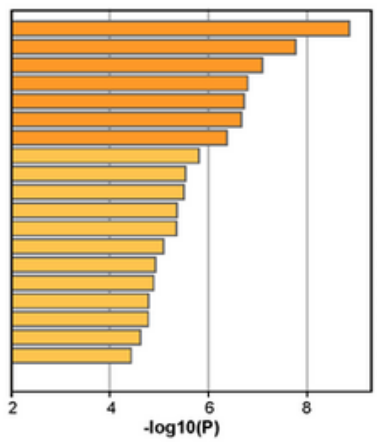

60:0030036: actin cytoskeleton organization GO:0001944: vasculature development GO.0045296: cadherin binding GO:0001974: blood vessel remodeling GO:0019901: protein kinase binding GO:0045785: positive regulation of cell adhesion G0098562. postoplasmic side of membrane -0009611: response to wounding GO-0007265: Ras protein signal transductio GO-0005938: cell cortex proteolysis (O) 0:0007169: transmembrane receptor protein tyrosine knase signaling path G0:0032990: cell part morphogenesis (

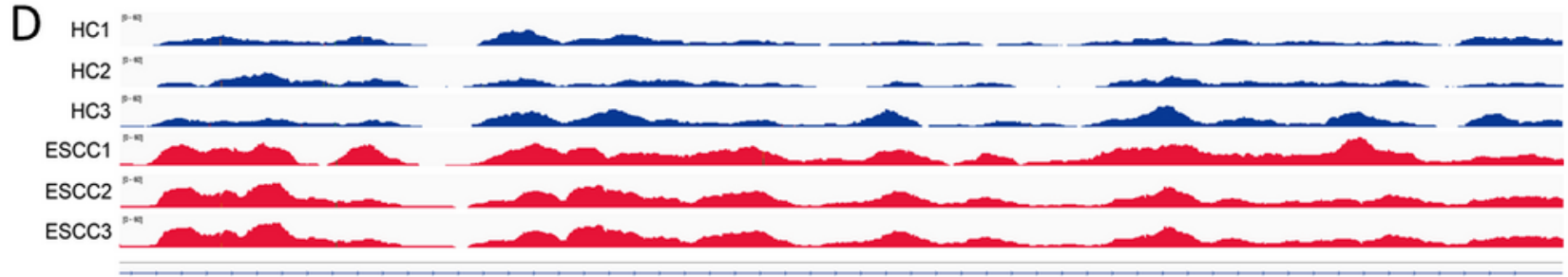

FOXK1-chr7:4,744,767-4,750,767

$E$

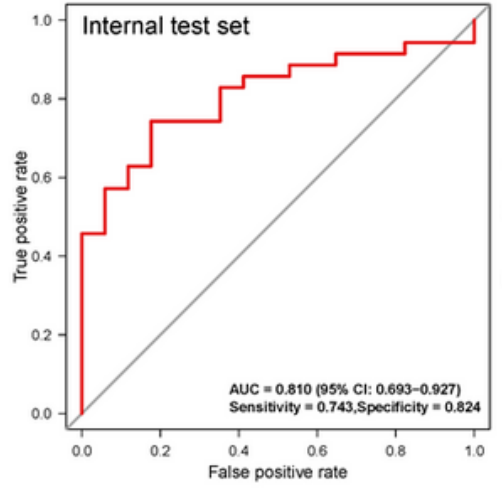

$\mathrm{F}$

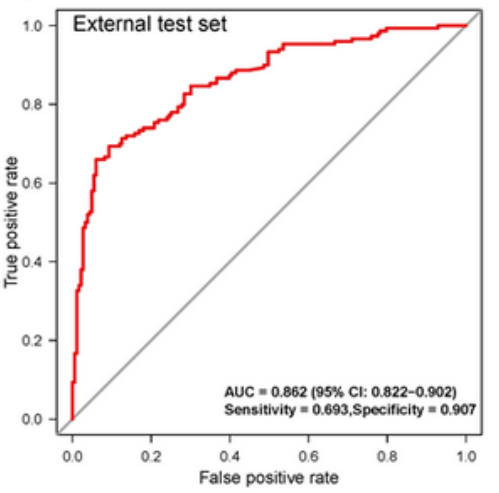

KEGG pathway

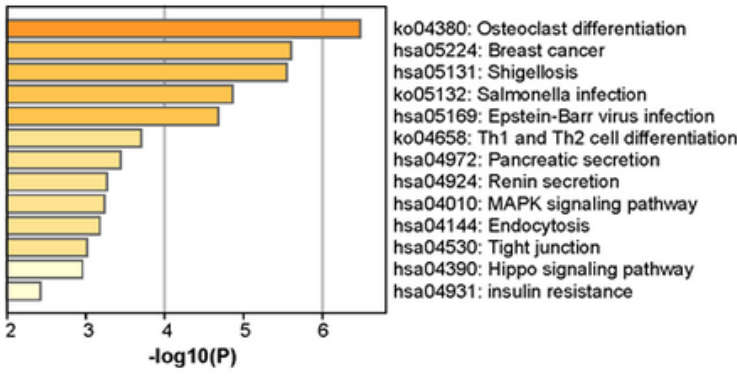

\section{Figure 3}

\section{Development, validation and performance of $5 \mathrm{hmC}$ diagnostic model.}

(A) Unsupervised hierarchical clustering of $71 \mathrm{HC}$ and $100 \mathrm{ESCC}$ cfDNA samples based on top $273 \mathrm{hmC}$ marker genes. 
(B) PCA plot of $71 \mathrm{HC}$ and $100 \mathrm{ESCC}$ cfDNA samples based on top $273 \mathrm{5hmC}$ marker genes.

(C) GO enrichment (left) and KEGG pathway enrichment (right) analysis of 273 biomarkers of the $5 \mathrm{hmC}$ classifier.

(D) The normalized $5 \mathrm{hmC}$ values of FOXK1 in HC and ESCC samples. (E-F) ROC curves and associated AUC values in the internal test set $(E)$ and the external test set $(F)$.

(G) Predictive probability scores based on $5 \mathrm{hmC}$ classifier for different clinical stages of internal test set samples.

$\mathrm{HC}$, healthy controls; ESCC, esophageal squamous cell carcinoma; FOXK1, forkhead box K1; ROC, receiver operating characteristic; AUC, area under curve; 5hmC, 5-hydroxymethylcytosines. 
A

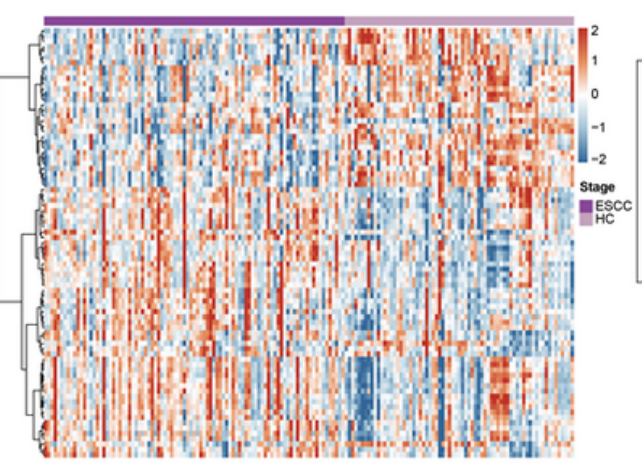

D

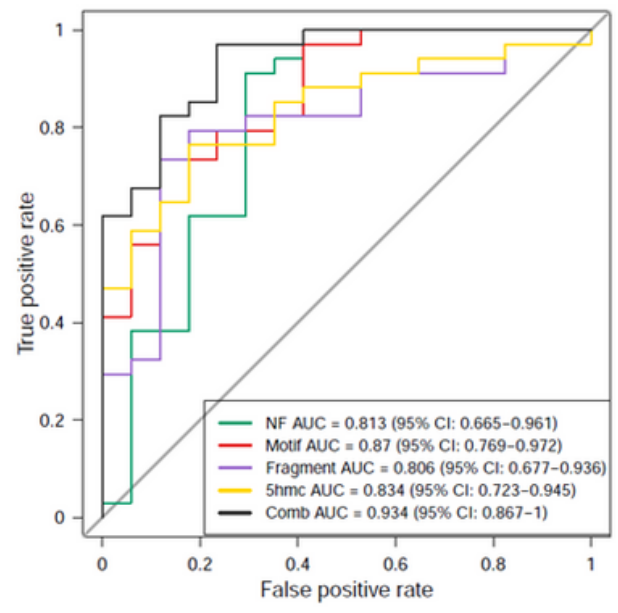

B

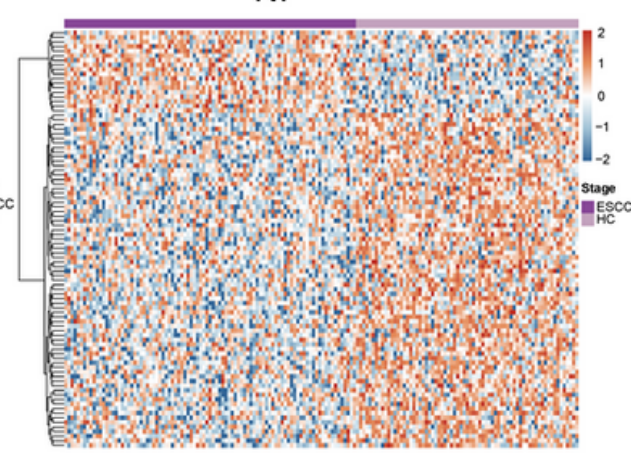

$\mathrm{E}$

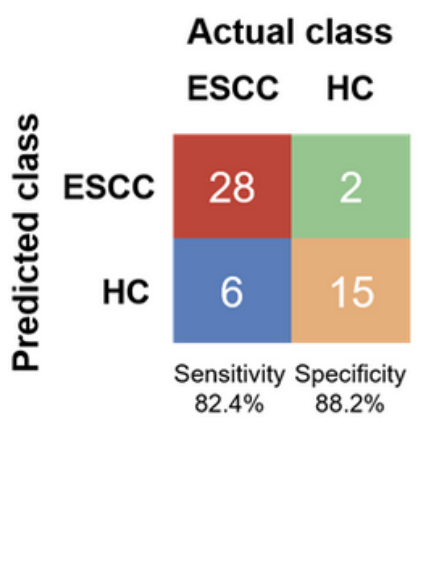

C

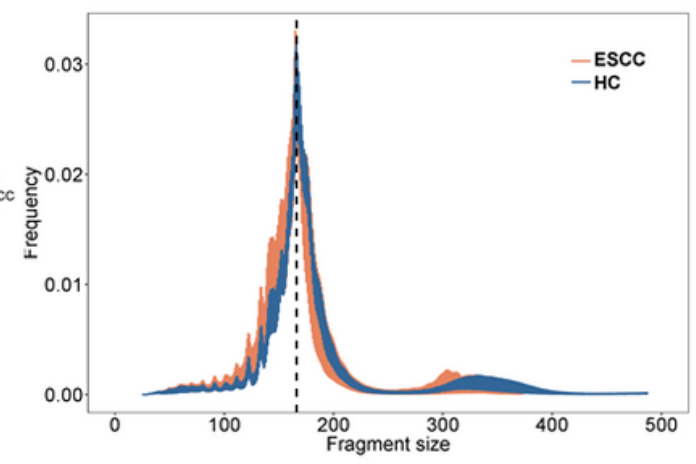

$\mathrm{F}$

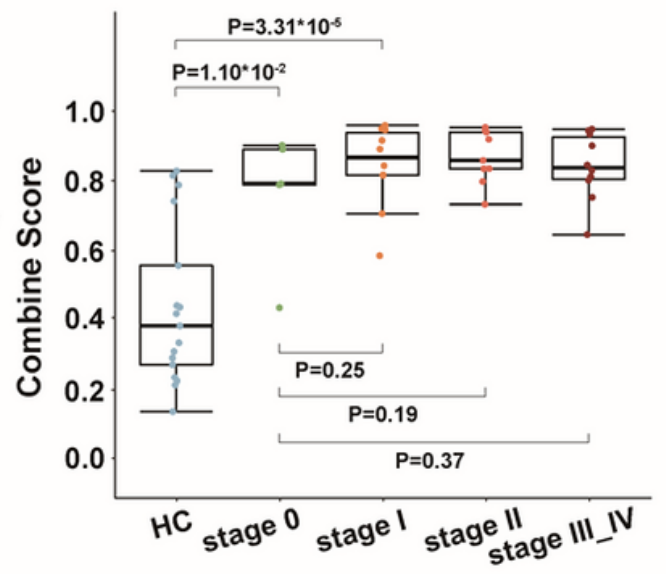

G

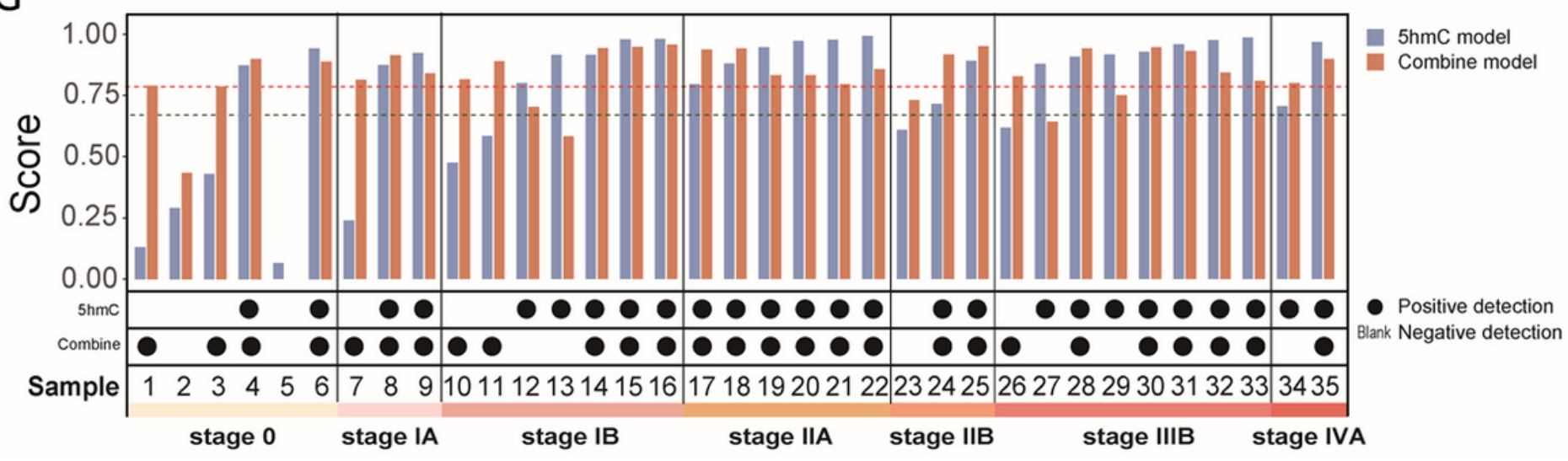

Figure 4

Development, validation and performance of the integrated diagnostic model.

(A) Heatmap analysis of differential motifs ( $p$ value $<0.001)$ between ESCC and HC samples.

(B) Heatmap analysis of genes with differential reads coverage between gene promoter and background regions ( $p$ values $<0.001$ ) in ESCC and $\mathrm{HC}$ samples.

(C) Frequencies comparison of different fragment sizes between ESCC and HC samples. 
(D) ROC curves and associated AUC values in the test set.

(E) Confusion matrices of integrated diagnostic model comparing the actual class with the predicted class for $\operatorname{ESCC}(n=34)$ and $\operatorname{HC}(n=17)$ samples in the test set.

(F) Predictive probability scores based on integrated diagnostic model for different clinical stages of the test set samples.

(G) Comparison of diagnostic performance between $5 \mathrm{hmC}$ model (blue) and integrated model (red) on different clinical stages of the test set ESCC samples $(n=35)$. The blue and red dotted line represent the thresholds of diagnostic positive of the $5 \mathrm{hmC}$ model and the integrated model, respectively. Positive ESCC detection is indicated by black dots, negative ESCC detection indicated by blank.

$\mathrm{HC}$, healthy controls; ESCC, esophageal squamous cell carcinoma; ROC, receiver operating characteristic; AUC, area under curve; $5 \mathrm{hmC}$, 5-hydroxymethylcytosines.

\section{Supplementary Files}

This is a list of supplementary files associated with this preprint. Click to download.

- SupplementaryLegendsandfiguresfinal.pdf

- TableS1Clinicopathologicalcharacteristics.xIsx

- TableS2Thetop10significantlyenrichment5hmCmotifs.xlsx

- TableS3Listofannotated5hmCmarkergenesinmodel.xlsx

- TableS4ListofWGSfeaturesinmodel.xIsx 